\title{
THE DISTANCES OF THE MAGELLANIC CLOUDS
}

\author{
ALISTAIR R. WALKER \\ Cerro Tololo Inter-American Observatory, NOAO, \\ Casilla 603, La Serena, Chile
}

\begin{abstract}
.
The present status of our knowledge of the distances to the Magellanic Clouds is evaluated from a post-Hipparcos perspective. After a brief summary of the effects of structure, reddening, age, and metallicity, the primary distance indicators for the Large Magellanic Cloud are reviewed: The SN 1987A ring, Cepheids, RR Lyraes, Mira variables, and Eclipsing Binaries. Distances derived via these methods are weighted and combined to produce final best estimates for the Magellanic Clouds distance moduli.
\end{abstract}

\section{Introduction}

The distances of the Magellanic Clouds (MC), in particular that for the Large Magellanic Cloud (LMC), are of great importance for three principal reasons: Firstly, the MC are sufficiently populous that they contain many different types of distance indicator, and are close enough so that most can be measured with high accuracy, thus they are invaluable for consistency comparisons. Secondly, the MC are sufficiently remote so that to first order the constituents of each may be taken to be at constant distance from us. Finally, the MC are a sanity check, for if we cannot agree upon the distance to two galaxies that are only a few 10's of kpc distant from us, how can we be sure of distances to more remote galaxies?

For these reasons the literature discussing MC distances is large. Chapter 2 of Westerlund (1997) comprehensively summarizes the subject to that date, somewhat disconcertingly he finishes with the statement In view of all the problems involved in the distance determinations it is necessary to admit that the distances of the two Clouds are still not sufficiently well known. In this chapter we will investigate whether or not the situation has changed for the better in the intervening two years, during which time Hipparcos 
astrometry has become available and photometry of massive numbers of stars as a byproduct of microlensing surveys is appearing in the literature.

We will begin (2) by reviewing some properties of the MC relevant to the present investigation, follow (3) by considering various distance indicators and conclude (4) by summarizing the present status of the MC distances together with some indication of possible future improvements. Almost all of the standard candles useful for MC distances rely on a galactic calibration, which for many of them will be discussed elsewhere in this volume.

\section{Relevant Properties of the Magellanic Clouds}

\subsection{STRUCTURE}

The LMC is a barred spiral, and defines the Magellanic subclass (SBm), (de Vaucouleurs \& Freeman 1972, Wilcots et al. 1996). Kinematical studies (Olszewski et al. 1991, Schommer et al. 1992) show that even the oldest populations (eg globular clusters containing RR Lyraes) have disk kinematics and there is presently no evidence for a pressure-supported halo (Olszewski, Suntzeff \& Mateo 1996). Thus for the LMC a satisfactory assumption is that most constituents are confined close to a well-defined disk that is only mildly tilted with respect to the plane of the sky.

However both the LMC and especially the Small Magellanic Cloud (SMC) show evidence for interactions between each other and the Galaxy. The extension of the SMC is very considerable in the line-of-sight (Gardiner \& Hatzidimitriou 1992) thus, unless the particular component of the SMC corresponding to a potential distance indicator can be unambiguously identified, it is of little use for finding the mean distance to the SMC. Consequently most of what follows pertains to the LMC rather than the $\mathrm{SMC}$, and perhaps the major utility of the SMC is as an aid in testing the metallicity sensitivity of distance indicators.

\subsection{REDDENING}

The main bodies of the $\mathrm{MC}$ are between galactic latitudes $-29^{\circ}$ to $-35^{\circ}$ (LMC) and $-43^{\circ}$ to $-46^{\circ}$ (SMC), and foreground reddening is expected to be non-negligible, especially for the LMC. Tanvir (1996) emphasizes that for certain distance scale applications having specific knowledge of the reddening can be circumvented, while in addition reddening corrections in the infrared are much less significant than in visual passbands. Bessell (1991) re-evaluated the foreground and internal reddening for both the SMC and LMC. He concludes that the foreground reddening for the SMC shows little variation and probably lies between $E(B-V)=0.04$ and 0.06 , while the foreground to the LMC is more varied, $E(B-V)=0.04$ to 0.09 . 
Average reddening within the $\mathrm{SMC}$ is about $E(B-V)=0.06$, with a similar figure for the LMC but he finds for the latter that there is a wider range of values than for the SMC. More recent observations for the LMC confirm this picture. Schlegel, Finkbeiner \& Davis (1998) present a full-sky galactic extinction map that is twice as accurate as the much-used Burstein \& Heiles (1982) map in regions of low to moderate reddening. The MC (and M31) are not removed from the new map but unfortunately accurate reddenings through these galaxies is not possible. The typical foreground reddening, measured from dust emission in surrounding annuli, is $E(B-V)=0.075$ for the LMC and $E(B-V)=0.037$ for the SMC. Noteworthy is that the new map is offset, with 0.02 mag higher reddening in high galactic latitudes, compared to Burstein \& Heiles (1982).

A major study of the reddening foreground to the LMC is that of Oestreicher, Gochermann \& Schmidt-Kaler (1995), who from UBV colors of 1409 galactic stars derive a reddening map with resolution 10 arcmin. The mean reddening is $E(B-V)=0.06 \pm 0.02$, however the distribution appears quite clumpy with a range from $E(B-V)=0.0$ to 0.15 . The higher values are interpreted as corresponding to dust clouds in the solar vicinity, these project to diameters typically 30-60 arcmin.

The reddening internal to the LMC is treated by Oestreicher \& SchmidtKaler (1996) from UBV photometry and spectral classifications. Their map of the reddening distribution correlates quite well with the HI column density (Luks \& Rohlfs 1992) and the IRAS 25 micron emission map (Israel \& Schwering 1986). The highest reddening occurs in the regions of 30 Doradus and the supershell LMC 2, reaching a maximum of $E(B-V)=0.29$, and stars in the bar are in general more highly reddened than elsewhere. However there are highly reddened stars spread out over the LMC, and conversely stars in the bar with rather low reddening. Some of the former stars, all of very high luminosity, may be reddened by circumstellar material, while some proportion of the latter stars may be located on the near side of the bar and thus suffer little internal LMC reddening. They also find that there are strong selection effects in that their intrinsically fainter stars $\left(V_{0}>13.3\right)$ show low reddenings, with median approximately $E(B-V)=0.07$.

The general conclusions are that:

1) Magnitude-limited samples are going be the lower-reddened stars.

2) The reddening is patchy enough, both galactic and in the LMC, that proceeding on a star by star or cluster by cluster basis seems prudent, if it is possible. In this context, an important program is that of Madore, Freedman \& Pevunova (in preparation) where OB star reddenings are being determined in the line of sight to many of the LMC Cepheid calibrators. This will allow direct comparison with the semi-empirical reddenings de- 
termined by Caldwell \& Coulson (1986) for most of these stars.

3) The more outer parts of the LMC suffer only galactic foreground reddening, as evinced by reddenings found for several LMC clusters containing RR Lyraes (Walker 1992).

4) The median reddening for stars in the $\mathrm{LMC}$ is $E(B-V) \sim 0.10$ and for those in the SMC probably slightly less, $E(B-V) \sim 0.08$.

5) With $A_{K} \sim 0.03$ on average, the advantages of working in the infrared are obvious.

\subsection{AGE, METALLICITY}

Most of the distance estimates for the MC rely on comparing a sample of stars in the MC with a corresponding galactic sample for which we know individual distances. In general, as might be expected, the galactic samples are rather better defined in terms of age and metallicity than are the MC samples. In some cases, for example Cepheids in galactic open clusters, the calibrating sample is rather sparse. Stated in general terms, we first need to have a reliable calibration for the galactic sample of stars, then we need to make the relevant observations for the MC sample, then we have to determine any differences between the samples and apply a correction if this affects the distance. Obviously, any direct (eg geometric) distance measurement circumvents these problems. Two illustrative examples follow.

Firstly, the sizeof the metallicity dependency of the Cepheid PeriodLuminosity (PL) relation has been controversial for several years (Madore \& Freedman 1991, Gould 1994a). Observational data relevant to the question include metallicities for galactic Cepheids in open clusters (Fry \& Carney 1997), for MC Cepheids (Luck et al. 1998), discussions of Cepheid data from microlensing project databases (Beaulieu et al. 1995), and the Hubble Space Telescope (HST) extra-galactic Cepheid surveys Freedman et al. 1994, Saha et al. 1994). These new results have been analyzed (Sasselov et al. 1997, Kochanek 1997, Kennicutt et al. 1998) with particular attention devoted to the $\mathrm{V}$ and I bands used for both HST surveys, where typically (Kennicutt et al. 1998) an effect on Cepheid distance moduli from $V$ and $I$ PL relations is $\sim-0.25 \pm 0.25 \mathrm{mag} / \mathrm{dex}$, although at times a stronger dependence has been suggested (Gould 1994a, Sekiguchi \& Fukugita 1998). With mean $[\mathrm{Fe} / \mathrm{H}]=-0.34(\sigma=0.15, n=32)$ for the LMC, and $[\mathrm{Fe} / \mathrm{H}]=-0.68(\sigma=$ $0.13, n=25$ ) for the SMC (Luck et al. 1998), the metallicity corrections are not large, particularly for the LMC. These Cepheid metallicities are in the mean more metal poor than earlier empirical or semi-empirical estimates (eg Caldwell \& Coulson 1986, Laney \& Stobie 1994), where $[\mathrm{Fe} / \mathrm{H}]=-0.15$ was adopted for the LMC. Note that a change from $[\mathrm{Fe} / \mathrm{H}]=-0.15$ to -0.3 will change the mean reddening for the LMC found by Caldwell \& 
Coulson (1986) from $E(B-V)=0.074$ to 0.059 , with consequent affect on use of this data for determining distances (Feast 1998).

Secondly, a new distance indicator, I magnitudes of red clump stars, has been developed by Paczyński and Stanek (1998). This can be directly calibrated from Hipparcos parallaxes, from which the mean absolute magnitude of 228 solar neighborhood red clump stars is found to be $M_{I}=-0.23 \pm 0.03$. Local group galaxies distances can thus be directly calibrated from Hipparcos results in a single step. Udalski et al. (1998) find MC distance moduli of 18.56 and 18.08 for the SMC and LMC respectively, with very small statistical errors (0.03 mag). Despite the seemingly photometrically well-defined populations of clumps stars locally and in the MC, these MC distance moduli are disconcertingly short compared to all other indicators. Cole (1998) suggests that a luminosity dependence of the red clump stars on both age and metallicity may resolve the apparent discrepancy, and revises the distances to the LMC and SMC to $18.36 \pm 0.17$ and $18.82 \pm 0.20$ respectively. Similar results are found by Giraldi et al. (1998). This method has much potential, once age and metallicity dependence effects have been definitively settled, given the ubiquitous nature of red clump stars and consequent small statistical errors in ensemble mean magnitudes.

\section{MC Distance Calibrations}

\subsection{A DIRECT DISTANCE TO THE LMC - THE SN 1987A RING}

Panagia et al. (1991) were the first to determine a direct distance to supernova (SN) 1987A by comparing high-accuracy measurements of the angular and physical size of the circumstellar ring surrounding the SN. The method is conceptually simple; the physical size can be calculated by measuring the light travel time to the ring, derived from International Ultraviolet Explorer (IUE) lightcurves of UV emission lines (eg NIII], NIV], NV, CIII]) observed between days 8 and 700 after the explosion, while the angular size is directly measured from HST images (eg O[III]) of necessity taken at later epochs. As might be expected, several assumptions must be made, and differing interpretations of the data are possible. The assumptions can be listed as:

1) The IUE lightcurves and the more recent images both correspond to gas that is in the same physical location.

2) The structure visible is a ring, and not some more complicated geometry.

3) The caustics in the IUE light curves do indeed represent the extreme light travel times.

4) The ring is circular and smooth.

5) The delay time between when the UV pulse first hit the ring and the appearance of the UV line emission is negligibly small. 
In general, quoted errors have reflected fitting errors to the observational data and have not attempted to realistically account for systematic effects arising from incorrect assumptions or inadequate models.

Gould (1994b) discussed assumptions 2), 3), and 4) in some detail, showing that the first two did indeed appear to be valid, and that ellipticity had little effect on the distance. Crotts, Kunkel, \& Heathcote (1995) confirmed the ring structure mostly from light-echo data. It should be noted that proof that the ring is circular will be tested when the SN shock hits it, a process that appears to be commencing (Sonneborn et al. 1998). Assumption 5) is generally thought to be valid, but if the delay time is accepted to be indeterminant then the derived distance becomes an upper limit rather than an equality (Gould 1995). The initial assumption 1) has been modeled by Lundquist and Sonneborn (1997), again a conservative interpretation of their results gives only an upper limit to the distance. We shall now discuss some of these results in more detail.

All analyses must by necessity use some subset of the IUE light curves, which are relatively noisy due to the IUE entrance aperture including stars 2 and 3, whose continua dominated the signal. Hubble Space Telescope (HST) images of the ring were first obtained in August 1990 (Jakobsen et al. 1991) with the Faint Object Camera (FOC) and have continued to be taken up to the present, in particular with the Space Telescope Imaging Spectrograph (STIS) which permits spatially resolved spectroscopy and is ideally suited for study of the SN environment.

The initial analysis by Panagia et al. (1991) used the early HST images in $\mathrm{O}[\mathrm{III}]$ to derive the angular size of the ring (1.66 \pm 0.03 arcsec, Jakobsen et al. 1991), and fitted a simple model to the time evolution of the NIII], NIV], NV and CIII] lines observed by IUE. The model fit to the NIII] lines is reasonable, but is poorer for the other lines. The latter have fewer observations and lower $\mathrm{S} / \mathrm{N}$ than the NIII] data. The times at which the lines first appear was found, in the mean, to be $83 \pm 6$ days, and the maximum $413 \pm 24$ days, these times being simply related to the size and inclination of the ring. The inclination of the ring thus derived was found to be in excellent agreement with the observed elliptical appearance, assuming that the ring is close to circular. The estimated distance to SN 1987A was $51.2 \pm 3.1$ kpc.

Gould (1994b) re-analysed the data, using a slightly different formalism and applied a correction to the center of the LMC based on the assumption that the SN lies in the LMC disk. The distance to the SN he found was $53.2 \pm 2.6 \mathrm{kpc}$. Gould (1995), in a more radical re-analysis of the data, fitted the UV light curves with a model based on those developed by Dwek \& Felton (1992), thought to be more appropriate for a ring geometry. He used only the NIII] and NIV] lines. The angular size of the ring was taken 
from Plait et al. (1995), a value $3 \%$ higher than used previously. His fits to the UV light curves yield times $8 \%$ smaller than found by Panagia et al. (1991), $75 \pm 3$ days and $390 \pm 2$ days. These two changes both act to reduce the derived distance. If assumption 5) above is correct then the distance to the $\mathrm{SN}$ is $46.7 \pm 0.7 \mathrm{kpc}$.

Sonneborn et al. (1997) perform a re-reduction of the IUE UV data, to provide what are likely to be definitive light curves. They also determined caustic timings of $84 \pm 4$ and $399 \pm 15$ days. Lundqvist \& Sonneborn (1997) re-analyse the ring geometry in detail, using recent [OIII] and [NII] HST images. In their models, together with those of Lundqvist \& Fransson (1996) they examine assumption 1) above, to conclude that the innermost parts of the [NII] emitting zone best represent the gas which emitted the UV lines. The corresponding angular radius of the inner edge of the [NII] emitting zone may be as small as $775 \pm 10$ mas, and this, together with their UV light curve timings from Sonneborn et al. (1997) gives an upper limit to the distance of the SN of $54.2 \pm 2.2 \mathrm{kpc}$.

Panagia et al. (1997), who use the new reductions of the IUE light curves by Sonneborn et al. (1997), and an extensive set of HST images, repeat and improve upon their earlier analysis and derive an absolute size of the ring $R_{a b s}=(6.17 \pm 0.18) 10^{17} \mathrm{~cm}$ and an angular size $R_{a n g}=808 \pm 17$ mas, to find a distance to the SN of $50.9 \pm 1.8 \mathrm{kpc}$.

Gould and Uza (1998), repeat the earlier analysis (Gould 1995) and also adopt the Sonneborn et al. (1997) re-evaluation of the UV data. They find shorter times for the caustic crossings, $80.5 \pm 1.7$ days and $378.3 \pm 4.8$ days but with less convincing fits than before. With an ellipticity estimate for the ring of $0.95 \pm 0.02$ from $\mathrm{A}$. Crotts, they derive a distance for the SN of $48.8 \pm 1.1 \mathrm{kpc}$, again pointing out that if assumption 5) is invalid then this becomes an upper limit. They also consider that the scenario of the initial UV and present optical emission lines coming from different zones is implausible, but note that such an effect could increase the distance modulus by up to $8 \%$, this conclusion in part based on the early Plait et al. (1995) (pre-CoSTAR) HST imaging data.

It is certain that our knowledge of the ring structure will increase dramatically as the ring is illuminated by passage of the SN shock front, via analysis of HST images and spectroscopy. More sophisticated models, both of the ring structure and of the energy distribution at break-out of the EUV radiation, should allow a consistent interpretation of all the IUE data and give confidence that we understand the ring structure. By contrast, the very careful re-reduction of the IUE data by Sonneborn et al. (1997) is unlikely to be improved upon, and thus represents a basic limitation to the timing accuracy of the caustic crossings. At this time it is clear that the interpretation of the present observational data sets (Plait et al. 1995, Panagia et 
al. 1997, Lundqvist \& Sonneborn 1997, Gould \& Uza 1998) have still not converged. The scatter of points near the peak of the N III] light-curve (see Gould \& Uza 1998 Figure 2) compared to various fitted models gives little confidence that the true position of the second caustic crossing is known to better than a value $390 \pm 15$ days. If the possible systematic effects 1 ) and 5) above are not significant then the Panagia et al. (1997) value for the SN distance of $50.9 \pm 1.8 \mathrm{kpc}$ should be close to the true value. If the situation is more complex than they assume then systematic effects could move this distance by up to $4 \mathrm{kpc}$ either way.

A correction to the rotation center (McGee \& Milton 1966, Bessell, Freeman \& Wood 1986) of the LMC is necessary. With rotation center at $\alpha(1950)=5 \mathrm{~h} 21 \mathrm{~m}, \delta(1950)=-69^{\circ} 18^{\prime}$, PA of line of nodes $171^{\circ}$, and inclination $27^{\circ}$, the plane of the LMC at the position of SN 1987A is 700 pc closer to us than the LMC center. Xu, Crotts \& Kunkel (1996) from a light-echo analysis show that the large complex of young stars and gas, LH 90 and $\mathrm{N} 157 \mathrm{C}$, lies $\sim 500 \mathrm{pc}$ in front of SN1987A, and it seems reasonable to suppose that the former lies very close to the plane, although recent HI absorption studies (Dickey et al. 1996) indicate that the velocity structure identified with the disk lies at least partially in front of 30 Doradus. Spyromilio et al. (1995) by contrast, argue from a light-echo analysis of $3 \mathrm{yr}$ of AAT plates that the N157C bubble lies behind the SN, and given that the echos represent material in front of the SN then the latter must lie close to the plane. Panagia et al. (1991) evaluate HI radial velocity structure (McGee \& Milton 1966, Radhakrishnan et al. 1972) and interstellar absorption components (Blades 1980) in the direction of 30 Doradus, and conclude that approximately two-thirds of the main body of the LMC is in front of SN 1987A. With the thickness of the LMC disk taken as 600 pc, this distance equal to the scale height of the older stars (Freeman, Illingworth \& Oemler 1983) then SN 1987A is $~ 100$ pc behind the plane. Despite these uncertainties in the location of the SN with respect to the LMC plane, the correction is not a large one. We will adopt a position for the $\mathrm{SN}$ of $300 \pm 200 \mathrm{pc}$ behind the plane and thus $400 \pm 200 \mathrm{pc}$ closer to us than the LMC center. The LMC distance modulus from this method is therefore $18.55 \pm 0.07$ (random) \pm 0.16 (systematic).

\subsection{CEPHEID DISTANCES}

The use of Cepheids as extragalactic distance indicators has recently been comprehensively reviewed by Tanvir (1996). Cepheid distances to the MC are traditionally found by comparing PL (period luminosity) or PLC (period luminosity color) relation zeropoints between the $\mathrm{MC}$ and our galaxy (Feast \& Walker 1987, Laney \& Stobie 1994). The galactic calibration can 
be via Cepheids in open clusters and associations, Cepheids with BaadeWesselink distances (Gieren, Fouqué, \& Gomez 1997) or via Cepheids with Hipparcos parallaxes (Feast \& Catchpole 1997, Madore \& Freedman 1997).

\subsubsection{Period Luminosity relation}

There are advantages to specifying the slope and zeropoint of the Cepheid period luminosity in the infrared, where the narrower width of the instability strip produces a tighter PL relation, pulsation amplitudes are smaller, and effects of reddening less, when compared to visual bandpasses. The galactic calibration has traditionally proceeded from a zero age main sequence (ZAMS) calibration of the few galactic clusters and associations containing Cepheids, with slope defined from the LMC Cepheids and zeropoint ultimately anchored to the distance to the Hyades. In recent times the zeropoint calibration has also been referenced to the Pleiades, with distance derived from a fit of the Pleiades ZAMS to local parallax stars (van Leuuwen 1983), on the grounds that the Pleiades is more similar in age and metallicity to the Cepheid-containing clusters. With the controversy over the Pleiades parallax as measured by Hipparcos (van Leeuwen \& Hansen Ruiz 1998, Pinsonneault et al. 1998, Soderblom et al 1998) it seems wisest at present to remain with a Hyades-based zeropoint. Hipparcos has determined a very accurate Hyades modulus of $3.33 \pm 0.01$ mag (Perryman et al. 1997) which so-happens to agree exactly with the mean of all ground-based Hyades distance measurements made in the past 20 years. The often quoted Feast \& Walker (1987) Cepheid ZAMS calibration was based on a Hyades modulus of 3.27, so their distance scale needs to be moved 0.06 mag more distant, thus corresponding to an LMC modulus of 18.53. A more recent discussion of the galactic ZAMS method by Laney \& Stobie (1994), where $\mathrm{PL}$ relations are derived in $\mathrm{J}, \mathrm{H}$ and $\mathrm{K}$ as well as $\mathrm{V}$, would move the distance scale only $0.02 \mathrm{mag}$ longer than this, if in both cases the Cepheids in associations are weighted half those in clusters and the same zeropoint is used, ie a LMC modulus of $18.55 \mathrm{mag}$, and $18.96 \mathrm{mag}$ for the SMC.

The PL relation can also be calibrated directly using Hipparcos parallaxes of field Cepheids. Unfortunately with the exception of a single star (Polaris) these parallaxes all have large relative errors. Feast and Catchpole (1997) combine parallaxes for 26 Cepheids, and determine a best fit $V$-band PL relation with zeropoint error \pm 0.10 mag. With LMC Cepheid photometry from Caldwell \& Laney (1991), reddening of $E(B-V)=0.074$ and a metallicity correction of $+0.042 \mathrm{mag}$, they derive an LMC distance modulus of $18.70 \pm 0.10$ mag. As a caveat, the mean parallax of these 26 stars is only $2.1 \mathrm{~ms}$ arc. Although the systematic error in the Hipparcos parallaxes is thought to be $\pm 0.1 \mathrm{~ms}$ arc (Brown et al. 1997), only a five percent effect for the Cepheids, treatment of systematic effects at this level 
requires extraordinary care, and some caution in evaluation of the results.

Madore \& Freedman (1998) compare the multi-wavelength LMC PL data (Madore \& Freedman 1991) with BVIJHK photometry for the Hipparcos Cepheids, unfortunately only seven stars have mean magnitudes available in all six bands, thus the results are not very robust given the large parallax errors for the galactic calibrators. Discounting their BV solution, which has very large error, and taking the mean of the remaining four solutions for various passband combinations, their LMC modulus is $18.54 \pm 0.12$. Feast (1998) cautions that biases may be introduced by grouping the data in this way.

Fitting PL relations to MC data relies on having a large body of wellcalibrated light curves for the MC Cepheids. The microlensing projects have produced high quality light-curves for very large numbers of Cepheids, the potential of which goes far beyond the use of Cepheids as distance indicators. However data in other important bandpasses (eg I, Tanvir 1996) and in the infrared is less extensive than desirable. In particular, Cepheids in the rich $\mathrm{MC}$ clusters await definitive observations, although programs are now beginning to address these needs (Ripepi 1998; W. Gieren, private communication).

\subsubsection{The Baade-Wesselink method}

The Baade-Wesselink (BW) method (Baade 1926, Wesselink 1946) and its variant, the Barnes-Evans method (Barnes \& Evans 1976), can in principle provide accurate distances to the MC Cepheids. A very thorough description of the method is given by Gautschy (1987), see also Balona (1977), and Feast \& Walker (1987), the difficulties are succinctly summarized by Paczyński (1996).

In recent years the method, which requires preferably simultaneous photometry and radial velocity measurements for the Cepheids, has moved to the infrared (Welch 1994, Laney \& Stobie 1995a,b, di Benedetto 1997, Gieren, Fouqué \& Gomez 1998), resulting in a reduction in both systematic and random errors (compare Figs 6 - 11 of Fouqué \& Gieren 1997). Laney \& Stobie (1995a) summarize the infrared advantages: the light variations at $\mathrm{K}$ are dominated by the change in surface area as opposed to temperature changes in the optical, infrared radius determinations are insensitive to whether the phases corresponding to the ascending branch are excluded, and the J-K or V-K color indices are insensitive to variations in microturbulence or surface gravity throughout the pulsation cycle.

Calibration of the surface-brightness relations can be provided by a combination of model atmospheres and a color-temperature calibration, but a direct calibration is preferable. It is now possible to use the many recent interferometric angular diameter measurements of nearby giants and su- 
pergiants, a substantial fraction of these measurements are being made in the infrared thus minimizing limb-darkening corrections. Fouqué \& Gieren (1997) have recently investigated in detail the applicability of the surface brightness - color relation for giants and supergiants to the Cepheids, and find excellent agreement with the slopes of the relations for all three types of star. Forcing the slope to be that for the Cepheids, they determine very precise zero-points for the near-infrared surface brightness - color relationships. Within the errors, these zeropoints agree with those found via the Cepheid effective temperature scale (Pel 1978) and from the lunar occultation diameter of $\zeta$ Gem (Ridgway et al. 1982).

Gieren et al. (1998) note that their period-radius relation is identical to that found by Laney \& Stobie (1995b) despite the use of very different methods. di Benedetto (1997) adopts a steeper period-radius relation (slope 0.73 cf 0.68), more recent evidence (Bono, Caputo, \& Marconi 1998) favors the shallower value.

Model atmosphere analyses (di Benedetto 1997, Bell \& Gustafsson 1989) show that the zero-point of the surface brightness - $(V-K)$ color relation is independent of metallicity to a level much less than 0.01 mag over a range of at least $0.5 \mathrm{dex}$ in $[\mathrm{Fe} / \mathrm{H}]$. Perhaps the major systematic uncertainty lies in the the $p$ factor applied to the integrated radial velocity curve in order to derive displacement, which is a correction for both geometric projection and limb darkening effects.

The results from all three recent investigations are in reasonable agreement. Gieren et al (1988) obtain an LMC modulus of 18.46 (they prefer to apply no metallicity correction, but a correction of +0.06 is their suggested value), Laney \& Stobie (1995b) find $18.58 \pm 0.04 \mathrm{mag}$ for the LMC and $19.00 \pm 0.04 \mathrm{mag}$ for the SMC, and di Benedetto (1997) derives $18.64 \pm 0.02$ for the LMC and 19.06 \pm 0.03 for the SMC, where for the latter two studies we have here increased the author's moduli by 0.06 so that the galactic cluster zeropoint corresponds to a Hyades modulus of 3.33 mag. The zeropoint error is not included in those above.

Rather than use infrared BW observations to calibrate a PL relation, measuring BW radii for the MC Cepheids directly would seem to be an attractive method for determining their distances in a very straight-forward manner, since such a procedure is essentially metallicity and reddening independent (Gieren et al. 1998). Such programs are underway, for both for Cepheids in MC clusters (W. Gieren, private communication) and in M31 and M33 (Stanek et al. 1998). LMC Clusters such as NGC 1866 and NGC 2031 each contain many Cepheids and with the advent of large-format IR imagers at least the imaging observations can be made at high efficiency. 


\subsection{RR LYRAES}

Udalski (1998) discusses distances to the MC and the galactic center based on observations made in the course of the Optical Gravitational Lensing experiment (OGLE). For 110 LMC and 128 SMC RR Lyraes he finds mean $\left.<I_{0}\right\rangle=18.41$ and 18.93 respectively, with errors for each estimated as $0.02 \mathrm{mag}$ (statistical) and $0.05 \mathrm{mag}$ (systematic). Absorption corrections are $A_{I}=0.33$ and 0.39 for the LMC fields, and $A_{I}=0.16$ in the SMC. Adopting a mean $(V-I)_{0}=0.45$ and 0.48 , then $\left\langle V_{0}\right\rangle=18.81$ and $\left\langle V_{0}\right\rangle=$ 19.41 for LMC and SMC respectively. Similarly, from the MACHO project database (Alcock et al. 1998) mean properties are found for a sample of 3454 RRab variables, the mean magnitude is $\left\langle V_{0}\right\rangle=19.00$. The difference $\Delta V_{0}=0.19$ mag between the two surveys could in principle almost all be due to the higher mean reddening adopted by Udalski (1998).

These results can be compared with the mean magnitudes of $\mathrm{MC}$ cluster RR Lyraes. For 182 RR Lyrae in seven LMC clusters, the mean $\left\langle V_{0}\right\rangle=$ $18.94 \pm 0.03$ (Walker 1992), while for four RR Lyraes in the SMC cluster NGC 121, Walker \& Mack (1988) find $\left\langle V_{0}\right\rangle=19.46 \pm 0.07$. The SMC comparison should not be over-interpreted given the uncertain location of NGC 121 with respect to the SMC center, although NGC 121 does appear to be located in a region of the SMC with relatively small depth in the line of sight (Gardiner \& Hatzidimitriou 1992). The mean metallicity of the cluster RR Lyraes is near $[\mathrm{Fe} / \mathrm{H}]=-1.9$ (Walker 1992) while that for the field stars is rather uncertain, for instance from a period-amplitude analysis Alcock et al. (1998) find a mean metallicity of $[\mathrm{Fe} / \mathrm{H}]=-1.2$, rather more metal rich than the canonical $[\mathrm{Fe} / \mathrm{H}] \sim-1.6$ often assumed. With a slope of $\sim 0.2$ for the RR Lyrae magnitude-metallicity relation (Fernley et al. 1998a) the cluster stars are expected to be $0.08-0.16$ mag brighter than the field stars on this basis. Given that the MC clusters with RR Lyraes have generous numbers of variables compared to the non-variable horizontal branch population, the majority of the cluster variables are expected from evolutionary lifetime arguments to be close to the ZAHB, and thus in this respect similar to the field population. Therefore the Walker (1992) and Alcock et al (1998) results seem consistent but the Udalski (1998) stars appear to be too bright by comparison.

To proceed further requires a calibration of the absolute magnitudes of RR Lyraes. This is controversial. Statistical parallax (Layden et al. 1996, Popowski \& Gould 1997, Fernley et al. 1998b) and Baade-Wesselink (Carney et al. 1992, Clementini et al. 1995) analyses of galactic field RR Lyraes find them fainter by typically $0.2-0.3$ mag than calibrations based on subdwarf parallaxes, (Gratton et al. 1997, Reid 1997, Pont et al. 1998), evolution theory (Caloi, D'Antona, \& Mazzitelli 1997) and pulsation theory 
applied to the double-mode (RRd) variables (Alcock et al. 1997a, Kovács \& Walker 1998). All the latter suggest an LMC modulus of $\sim 18.5$ mag. Catelan (1998) showed that galactic cluster and field RR Lyraes have the same distribution in the period-temperature diagram, and he argues that any a consequence of this result is that any difference in luminosity between the two groups of stars is very unlikely. The LMC results above further support this result. Only a single star, RR Lyrae itself, has a Hipparcos parallax of any significance, with a consequent $\pm 0.3 \mathrm{mag}$ error in its absolute magnitude (Fernley et al. 1997).

\subsection{MIRA VARIABLES}

The use of Mira variables as distant indicators is discussed in detail elsewhere in this volume. Occuring in the general field, in metal rich globular clusters, and in the galactic center, as well as being easily bright enough to be accurately measured in local group galaxies, they are an important complement to more traditional distance indicators such as the Cepheids and RR Lyraes. Infrared PL relations with small scatter have been found for Mira variables in the MC (Feast et al. 1989, Groenewegen \& Whitelock 1996). Wood (1995) found no strong evidence for a metallicity dependence, by comparing results for LMC and SMC Miras, but earlier Wood (1990) had suggested that $M_{K}$ should be less sensitive to metallicity effects than $M_{b o l}$. The zeropoint can be calibrated from Miras in metal rich globular clusters, these with distances by other means (eg RR Lyraes, main sequence (MS) fitting to local subdwarfs), from an assumed distance to the galactic center or, most directly, from Hipparcos parallaxes to a few nearby Miras. As with the Cepheids, there are few such stars in the catalog. Restricting the sample to the 11 oxygen-rich Miras with Hipparcos parallaxes, and defining the PL relation slope from the LMC Miras, a mean of the $M_{K}$ and $M_{b o l}$ relations gives an LMC modulus of $18.54 \pm 0.18$. If $M_{K}$ alone is to be preferred then the distance increases to $18.60 \pm 0.18$.

\subsection{DETACHED ECLIPSING BINARIES}

Paczyński (1996) has reviewed the use of detached eclipsing binary systems as distance indicators. He advocates the use of double-lined systems as distance indicators, applicable to galaxies throughout the Local Group. For the MC, many potential candidates have been identified from the microlensing surveys (eg Alcock et al. 1997b). Although intensive observing is then needed to obtain accurate photometry and radial velocities, the method has the advantage of being near-direct. If the selected binary is indeed welldetached and uncomplicated, then only a surface brightness - color relation is needed in order to calculate the distance, in addition to directly mea- 
sured quantities. This relation can be calibrated from interferometrically measured stellar angular diameters, and as for the Cepheids, it is probably best to use a color index such as $V-K$. Guinan et al. (1997) provide a preliminary report on results for the LMC eclipsing binary HV 2774, combining ground-based photometry and spectroscopy with HST spectroscopy. They find a distance modulus for the LMC of $18.54 \pm 0.08$ mag.

\subsection{OTHER METHODS}

Several other methods can provide distances to the MC. The tip of the red giant branch (TRGB), (Lee, Freedman \& Madore 1993, Madore, Freedman \& Sakai 1996) can be clearly defined given sufficient numbers of stars, and appears to be an excellent distant indicator for low-metallicity populations, particularly in an $I, V-I \mathrm{CMD}$ where the externally defined dispersion is less than $\pm 0.1 \mathrm{mag}$. Since it relies on the galactic globular cluster for calibration, the TRGB method is subject to the same distance scale uncertainties that plague the RR Lyrae distance scale.

The Planetary Nebulae (PN) luminosity function (Jacoby et al. 1992, Jacoby 1997) is calibrated by assuming a distance to M31. The Jacoby, Walker \& Ciardullo (1990) distance moduli for the MC, adjusting to the Freedman \& Madore (1990) M31 modulus, are $18.50 \pm 0.18$ for the LMC, based on $42 \mathrm{PN}$, and $19.15 \pm 0.29$ for the SMC, based on 8 PN. Since the M31 distance is based on a galactic Cepheid calibration, the PN do not provide independent zeropoints for the MC.

Bond (1996) describes the use of post asymptotic giant-branch (PAGB) stars as distance indicators. The galactic calibration on such stars in galactic globular clusters, and so again is tied to the RR Lyrae distance scale, together with its present calibration uncertainties. Given the rather few PAGB galactic calibrators, the main use of such stars in the MC is likely to be in strengthening the calibration, once a definitive distance to the $\mathrm{MC}$ can be found by other means.

Various luminosity calibrations of novae light curves have been presented by Della Valle \& Livio (1995) and Livio (1997), based mostly on novae in M31, but also include 15 LMC novae. Livio (1997) lists the several advantages of novae as distance indicators which includes their brightness, no metallicity dependence, good theoretical understanding, and rather small intrinsic scatter. Their discovery and consequent study is observationally intensive, and like the PN and PAGB stars, the use of the MC novae is more important in strengthening the galactic calibration by assuming $\mathrm{MC}$ distances from some other source.

Many color magnitude diagrams (CMDs) of MC clusters appear in the literature, and in the 15 years since the introduction of CCDs the resulting 
photometry has been accurate enough to allow distances to be derived either by comparison either with similar galactic clusters, assuming a distance for the latter, or with theoretical isochrones. The isochrone comparison has traditionally been used to determine all of age, distance, metallicity and reddening, a sufficient number of variables that it is near-impossible to test any of the assumptions (mixing-length, overshoot, etc.) used in building the isochrones. Far better is to measure metallicity and reddening separately so that the relevance of the chosen set of isochrones can be definitively tested. The many $\mathrm{MC}$ intermediate age clusters are obvious targets, the younger of these may be able to be compared directly to the Hyades, with suitable differential metallicity corrections. For most of the clusters, spectroscopic metallicities are not known. Available CMDs (see list in Westerlund 1997) have favored LMC distance moduli in the range $18.4-18.6$, early smaller moduli are mostly a consequence of incorrect isochrone color-temperature calibrations. The younger populous MC clusters, such as NGC 1866, are also prime targets, with the advantage that several contain significant numbers of Cepheid variables thus allowing a direct distance comparison. If CMDbased distances accurate to $<0.1 \mathrm{mag}$ are to be produced then the absolute photometric accuracy demanded of the observations is very high due to the steepness of the main sequence in the CMD. Dereddened colors of the MS should have error no larger than $\pm 0.02 \mathrm{mag}$, and preferably nearer to \pm 0.01 mag. It is doubtful whether any of the published CMD's have reached this level of accuracy, although it is certainly not an impossible task given sufficient attention to the calibration issues.

\section{CONCLUSIONS, AND THE FUTURE}

The Hipparcos mission has provided parallaxes for the traditional distance indicators such as Cepheids and Miras, enabled alternative calibrations for the open cluster route to Cepheid luminosities and via subdwarfs to Miras and RR Lyraes in globular clusters. It has also allowed the invention of new indicators, such as the red clump stars. All these distance indicators are present in the MC, where the luminosity scales can be compared directly. Although the mission was undeniably a great success, it is unfortunate that the accuracy limits to the Hipparcos parallaxes are such that the Cepheid, RR Lyrae and Mira distance scales cannot be fixed to the few percent accuracy that is scientifically so desirable.

Microlensing surveys have provided high quality optical photometry for many thousands of variables in the $\mathrm{MC}$, the galactic bulge and a few other selected targets. The great value of this photometry for the pulsating variables is that it provides statistically significant samples of stars with differing masses, temperatures, metallicities and pulsation modes in order to 
compare with evolution and pulsation theory. Our understanding of these stars will be greatly improved as a result, which should enhance their value as distance indicators.

Distance measurements that contain the minimum number of steps and assumptions will be those subject to the least number of systematic errors. The SN 1987A distance to the LMC is one that is independent of all other distance indicators, and as discussed above, more definitive quantification of possible systematic effects should be possible from observations made in the next few years. Eclipsing binaries have been discovered in profusion in the $\mathrm{MC}$ by the microlensing surveys, and detached double-lined systems can provide a near-direct distance estimate. The direct infrared Baade-Wesselink calibration of MC Cepheids is now also underway, with increased confidence that the systematic effects that have plagued the visual wavelengths version of the method are indeed under control.

The new large telescopes in the south (ESO VLT, Gemini, Magellan) together with new instrumentation, particularly that working in the nearinfrared, will play a large part in resolving MC distance concerns, with accurate observations of many of the distance indicators mentioned above, and will provide detailed spectroscopic analyses of MC populations in general. The move to the infrared has reduced dependence on reddening, but definitive calibrations of metallicity effects are not yet available.

Our present evaluation is that the center of the SMC has a modulus $0.42 \pm 0.05$ greater than that of the LMC, a result depending mostly on the Cepheids. It assumes for both LMC and SMC that the space distributions of the two sets of stars are not, in the mean, offset from the centers of the galaxies. A "best" distance for the LMC can be obtained either by taking a simple mean of all distance indicators, or else by suitable weighting of the indicators. Since systematic errors are likely to dominate in almost all cases, the former method is rather unsatisfactory due to the possible dominance of outliers, while the latter relies on ad-hoc weighting that is very difficult to quantify scientifically. The evaluation here is that calibrations based on galactic field RR Lyraes, RGB clump stars, and comparison of MC cluster CMD's to isochrones or galactic clusters, should be weighted zero at present. That leaves us with SN 1987A, Cepheids (via clusters, Baade-Wesselink, Hipparcos), RR Lyraes (via Hipparcos subdwarf calibration of Globular cluster distances), Miras, and an eclipsing binary. For these a mean modulus is $18.55 \pm 0.10 \mathrm{mag}$, where the error estimate is approximate and one which conservative readers versed in the history of the subject may well prefer to double. A different weighting scheme can produce very different results, for instance (Fernley et al. 1997), emphasising a calibration based on galactic RR Lyraes and discounting the distance indicators prefered here produces an LMC modulus near 18.3 mag. Until discrepancies such as this are sorted 
out we cannot be entirely sure that we have made the correct choice.

It is clear from the discussions in this chapter that we can only conclude, as did Westerlund (1997), that the distances to the MC are not yet sufficiently well-known, despite the success of the Hipparcos mission and the invaluable microlensing photometry. Further analyses of their results, and the results of on-going programs should over the next few years provide more definitive distances. In the longer term, beyond 2005, the Space Interferometry Mission (SIM, see http://sim.jpl.nasa.gov/sim/) is expected to provide parallaxes accurate to $4 \mu$ arcsec for 10000 stars, and which corresponds to a $5 \sigma$ measure for a star in the LMC, while the later GAIA mission (Lindegren \& Perryman 1996) will provide 50 million parallaxes with an accuracy of better than $10 \mu$ arcsec. These missions will certainly resolve all questions relating to distances within our galaxy, and reduce the uncertainty in the $\mathrm{MC}$ distances to below one percent.

\section{References}

Alcock, C., et al. 1997a, ApJ, 482, 89

Alcock, C., et al. 1997b, AJ, 114, 326

Alcock, C., et al. 1998, preprint

Baade, W. 1926, Astr. Nachr., 228, 359

Balona, L.A. 1977, MNRAS, 178, 231

Barnes, T.G., \& Evans, D.S. 1976, MNRAS, 174, 489

Beaulieu, J.P., et al. 1995, A\&A, 303, 137

Bell, R.A., \& Gustafsson, B. 1989, MNRAS, 236, 653

Bessell, M.S. 1991, A\&A, 242, L17

Bessell, M.S., Freeman, K.C., \& Wood, P.R. 1986, ApJ, 310, 710

Blades, J.C. 1980, MNRAS, 190, 33

Bond, H.E. 1997, in The Extragalactic Distance Scale, eds, Livio, M., Donahue, M., \& Panagia, N., publ. Cambridge Univ. Press, Cambridge, p.224

Bono, G., Caputo, F., \& Marconi, M. 1998, astro-ph/9802127

Brown, A.G.A., Arenou, F., van Leeuwen, F., Lindegren, L., \& Luri, X. 1997, in Proceedings of the Hipparcos, Venice 1997 Symposium, publ. European Space Agency, ESA-SP 402

Burstein, D., \& Heiles, C. 1982, AJ, 87, 1165

Caldwell, J.A.R., \& Coulson, I.M. 1986, MNRAS, 218, 233

Caldwell, J.A.R., \& Laney, C.D. 1991, in The Magellanic Clouds, IAU Symposium no. 148, eds. Haynes, R., \& Milne, D., publ. Kluwer, Dordrecht, p. 249

Caloi, V., D'Antona, F., \& Mazzitelli, I. 1997, A\&A, 320, 823

Carney, B.W., Storm, J., \& Jones, R.V. 1992, ApJ, 386. 663

Catelan, M. 1998, ApJ, 495, L81

Clementini, G., Carretta, E., Gratton, R., Merighi, R., Mould, J.R., \& McCarthy, J.K. 1995, AJ, 110, 2319

Cole, A.A. 1998, ApJ, in press, astro-phys/9804110

Crotts, A.P.S., Kunkel, W.E., \& Heathcote, S.M. 1995, ApJ, 438, 724

Della Valle, M., \& Livio, M. 1995, ApJ, 452, 704

de Vaucouleurs, G., \& Freeman, K.C. 1972, Vistas in Astron., 14, 163

di Benedetto, G.P. 1997, ApJ, 486, 60

Dickey, J.M., Duesterberg, C., Mebold, U., Mueller, H., Osterberg, J., Staverly-Smith, L. 1996, BAAS, 188, \#51.05 
Dwek, E., \& Felton, J.E. 1992, ApJ, 387, 551

Feast, M.W. 1998, MNRAS, 293, L27

Feast, M.W., \& Catchpole, R.M. 1997, MNRAS, 286, L1

Feast, M.W., \& Walker, A.R. 1987, Ann. Rev. Astron. Astrophys., 25, 345

Feast, M.W., Glass, I.S., Whitelock, P.A., \& Catchpole, R.M. 1989, MNRAS, 241, 375

Fernley, J., et al. 1997, in Proceedings of the Hipparcos, Venice 1997 Symposium, publ.

European Space Agency, ESA-SP 402

Fernley, J., Carney, B.W., Skillen, I., Cacciari, C., \& Janes, K. 1998a, MNRAS, 293, L61

Fernley, J., et al. 1998b, A\&A, 330, 515

Fouqué, P., \& Gieren, W.P. 1997, A\&A, 320, 799

Freedman, W.L., et al. 1994, ApJ, 427, 628

Freedman, W.L., \& Madore, B.F. 1990, ApJ, 365, 186

Freeman, K.C., Illingworth, G., \& Oemler, A. 1983, ApJ, 272, 488

Fry, A.M., \& Carney, B.W. 1997, AJ, 113, 1073

Gardiner, T.L., \& Hatzidimitriou, D. 1992, MNRAS, 257, 195

Gautschy, A. 1987, Vistas in Astron., 30, 197

Gieren, W.P., Fouqué, P., \& Gomez, M. 1998, ApJ, 488, 74

Giraldi, L., Groenewegen, M.A.T., Weiss, A., \& Salaris, M. 1998, MNRAS submitted, astro-ph/9805127

Gould, A. 1994a, ApJ, 426, 542

Gould, A. 1994b, ApJ, 425, 51

Gould, A. 1995, ApJ, 452, 189

Gould, A., \& Uza, O. 1998, ApJ, 494, 118

Gratton, R.G., Fusi Pecci, F., Carretta, E., Clementini, G., Corsi, C.E., Lattanzi, M.G. 1997, ApJ, 491, 749

Groenewegen, M.A.T., \& Whitelock, P.A. 1996, MNRAS, 281, 1347

Guinan, E.F., et al. 1987, BAAS, 191, \#03.13

Israel, F.P., \& Schwering, P.B. 1986, in Light on Dark Matter,ed. Israel, F.P., publ. Reidel, Dordrecht, p. 383

Jacoby, G.H., Branch, D., Ciardullo, R., Davies, R.L., Harris, W.E., Pierce, M.J., Pritchet, C.J., Tonry, J.L., \& Welch, D.L. 1992, PASP, 104, 599

Jacoby, G.H. 1997, in The Extragalactic Distance Scale, eds. Livio, M., Donahue, M., \& Panagia, N., publ. Cambridge Univ. Press, Cambridge, p. 197

Jacoby, G.H., Walker, A.R., \& Ciardullo, R. 1990, ApJ, 365, 471

Jakobsen, P., et al. 1991, ApJ, 369, L63

Kennicutt, R.C., et al. 1998, ApJ, 498, 181

Kochanek, C.S. 1997, ApJ, 491, 13

Kovács, G., \& Walker, A.R. 1998, ApJ, submitted

Laney, C.D., \& Stobie, R.S. 1994, MNRAS, 266, 441

Laney, C.D., \& Stobie, R.S. 1995a, MNRAS, 274, 337

Laney, C.D., \& Stobie, R.S. 1995b, In Astrophysical Applications of Stellar Pulsation, eds. Stobie, R.S., \& Whitelock, P.A., ASP Conf. Ser., 83, 254

Layden, A.C., Hanson, R.B., Hawley, S.L., Klemola, A.R., \& Hanley C.J. 1996, AJ, 112, 2110

Lee, M.G., Freedman, W.L., \& Madore, B.F. 1993, ApJ, 417, 553

Lindegren, L., \& Perryman, M.A.C. 1996, A\&AS, 116, 579

Livio, M. 1997, in The Extragalactic Distance Scale, eds. Livio, M., Donahue, M., \& Panagia, N., publ. Cambridge Univ. Press, Cambridge, p. 186

Luck, R.E., Moffett, T.J., Barnes, T.G., \& Gieren, W.P. 1998, AJ, 115, 605

Luks, T., \& Rohlfs, K. 1992, A\&A, 263, 41

Lundqvist, P., \& Fransson, C. 1996, ApJ, 464, 924

Lundquist, P., \& Sonneborn, G. 1997, in SN 1987A: Ten Years After, eds. Phillips, M., \& Suntzeff, N., ASP Conf. Ser., in press, astro-ph/9707144

Madore, B.F., Freedman, W.L., \& Sakai, S. 1997, in The Extragalactic Distance Scale, eds. Livio, M., Donahue, M., \& Panagia, N., publ. Cambridge Univ. Press, Cambridge, p. 
239

Madore, B.F., \& Freedman, W.L. 1991, PASP, 103, 933

Madore, B.F., \& Freedman, W.L. 1998, ApJ, 492, 110

McGee, R.X., \& Milton, J.A. 1966, Aust. J. Phys., 19, 343

Oestreicher, M.O., Gochermann, J., \& Schmidt-Kaler, T. 1995, A\&AS, 112, 495

Oestreicher, M.O., \& Schmidt-Kaler, T. 1996, A\&AS, 117, 303

Olszewski, E.W., Schommer, R.A., Suntzeff, N.B., \& Harris, H.C. 1991, AJ, 101, 515

Olszewski, E.W., Suntzeff, N.B., \& Mateo, M. 1996, Ann. Rev. Astron. Astrophys., 34, 511

Paczyński, B. 1997, in The Extragalactic Distance Scale, eds. Livio, M., Donahue, M., \& Panagia, N., Cambridge Univ. Press, Cambridge, p. 273

Paczyński, B., \& Stanek, K.Z. 1998, ApJ, 494, L189

Panagia, N., Gilmozzi, R., Macchetto, F., Adorf, K-M., \& Kirshner, R. 1991, ApJ, 380, L23

Panagia, N., Gilmozzi, R., Kirshner, R.P., Pun, C.S.J., \& Sonneborn, G. 1997, BAAS, 191, \#19.09

Pel, J.W. 1978, A\&A, 62, 75

Perryman, M.A.C., et al. 1998, A\&A, 331, 81

Pinsonneault, M.H., Stauffer, J., Soderblom, D.R., King, J.R., Hanson, R.B. 1998, ApJ submitted, astro-ph/9803233

Plait, P.C., Lundqvist, P., Chevalier, R.A., Kirshner, R.P. 1995, ApJ, 439, 730

Pont, F., Mayor, M., Turon, C., \& Vandenberg, D.A. 1998, A\&A, 329, 87

Popowski, P., \& Gould, A. 1997, ApJ submitted, astro-ph/9703140

Radhakrishnan, V., Brook, J.W., Gross, W.M., Murray, J.D., \& Schwarz, U.J. 1972, ApJS, 24, 1

Reid, I.N. 1997, AJ, 114, 161

Ridgway, S.T., Jacoby, G.H., Joyce, R.R., Siegel, M.J., \& Wells, D.C. 1982, AJ, 97, 680

Ripepi, V. 1998, in A Half-Century of Stellar Pulsation Interpretations, eds. Bradley, P.A., \& Guzik, J.A., ASP Conf. Ser., 135, 169

Saha, A., Labhardt, L., Schwengeler, H., Macchetto, F.D., Panagia, N., Sandage, A., \& Tammann, G.A. 1994, ApJ, 425, 14

Sasselov, D., et al. 1997, A\&A, 324, 471

Schlegel, D.J., Finkbeiner, D.P., \& Davis, M. 1998, ApJ, in press, astro-ph/9709041

Schommer, R.A., Olszewski, E.W., Suntzeff, N.B., \& Harris, H.C. 1992, AJ, 103, 447

Sekiguchi, M., \& Fukugita, M. 1998, Observatory, 118, 73

Soderblom, D.R., King, J.R., Hanson, R.B., Jones, B.F., Fischer, D., Stauffer, J.R. 1998, ApJ submitted, astro-ph/9801281

Sonneborn, G., et al. 1997, ApJ, 477, 848

Sonneborn, G., et al. 1998, ApJ., 492, 139

Spyromilio, J., Malin, D.F., Allen, D.A., Steer, C.J., \& Couch, W.J. 1995, MNRAS, 274, 256

Stanek, K.Z., Kaluzny, J., Krockenberger, M., Sasselov, D.D., Tonry, J.L., \& Mateo, M. 1998, AJ, 115, 1894

Tanvir, N.R. 1996, in The Extragalactic Distance Scale, eds. M. Livio, M. Donahue, N. Panagia, Cambridge Univ. Press, Cambridge, p. 91

Udalski, A. 1998, Acta. Astron., submitted, astro-phys/9805221

Udalski, A., Szymanski, M., Kubiak, M., Pietrzynski, G., Wozniak, P., \& Zebrun, K. 1998, Acta. Astron., 48, 1

van Leeuwen, F. 1983, Ph.D thesis, Leiden Univ., Neth.

van Leeuwen, F., Feast, M.W., Whitelock, P.A., \& Yudin, B. 1997, MNRAS, 287, 955

van Leeuwen, F., \& Hansen Ruiz, C.S. 1998, A\&AS, in press

Walker, A.R. 1992, Mem. S.A.It., 63, 479

Walker, A.R., \& Mack, P. 1988, AJ, 96, 872

Welch, D.L. 1994, AJ, 108, 1421

Wesselink, A.J. 1946, Bull. Astron. Inst. Neth., 10, 91 
Westerlund, B.E. 1997, The Magellanic Clouds, publ. Cambridge Univ. Press, Cambridge Wilcots, E.M., Lehman, C., \& Miller, B. 1996, AJ, 111, 1575

Wood, P.R. 1990, in From Miras to Planetary Nebulae, eds. Mennessier, M.O., \& Omont, A. Edition Frontières, Gif-sur-Yvette, p. 67

Wood, P.R. 1995, in Astrophysical Applications of Stellar Pulsation, eds. Stobie, R.S., \& Whitelock, P.A., ASP Conf. Ser. 83, p. 127

Xu, J., Crotts, A.P.S., Kunkel, W.E. 1995, ApJ, 451, 806 